\title{
Vers une approche physiopathologique des troubles de l'éjaculation
}

\author{
F. GIULIANO (1), K. MAC KENNA (2), S. DROUPY (1), O. RAMPIN (3), V. IZARD (1), G. BENOIT (1), \\ A. JARDIN (1) \\ [1) Service d'Urologie, CHU de Bicêtre, Le Kremlin Bicêtre \\ [2) Department of Physiology, NorthWestern University, Chicago, Illinois, USA \\ [3) Unité de Neurobiologie Végétative, INRA, Jouy en Josas
}

\section{RÉSUMÉ}

Les mécanismes locaux de l'éjaculation comprenant l'émission et l'expulsion du sperme sont commandés non seulement par la composante sympathique de l'innervation autonome mais également par l'innervation parasympathique auxquelles s'associe l'innervation somatique de la musculature striée périnéale empruntant le nerf pudendal. Les 2 phases de l'éjaculation sont médiées par des réflexes organisés aux étages spinaux thoraco-lombaires et sacrés dont l'organisation et la coordination demeurent mal connues. De nombreux neuromédiateurs périphériques participent à la survenue de l'éjaculation : les neuromédiateurs classiques : noradrénaline et acétylcholine mais également des neuromédiateurs non adrénergiques non cholinergiques c'est à dire peptidergiques, purinergiques et le monoxyde d'azote. Le rôle des afférences de l'ensemble tractus séminal dans le déclenchement de l'éjaculation n'a pas été exploré à l'exception de l'innervation de la verge représentée par le nerf dorsal de la verge. La participation de plusieurs centres supraspinaux à la modulation de l'activité des centres spinaux qui commandent l'éjaculation a été mise en évidence : des noyaux hypothalamiques, l'amygdale, et le noyau paragigantocellulaire. Sur la base de ces données anatomiques et neurophysiologiques, la physiopathologie des perturbations de l'éjaculation est ensuite discutée. L'éjaculation prématurée pourrait être liée à l'existence d'une hypersensibilité à la stimulation à la fois périphérique et centrale. Les autres troubles de l'éjaculation résultent d'al- térations des mécanismes de commande centraux et/ou périphériques.

Mots clés : Ejaculation, neurophysiologie, éjaculation prématurée, anéjaculation

\section{INTRODUCTION}

La connaissance biologique des fonctions sexuelles mâles s'est considérablement accrue ces dernières années. La recherche clinique et les expériences menées chez l'animal ont permis en particulier de mieux connaître les mécanismes locaux de l'érection et la physiopathologie de l'insuffisance érectile. Ces découvertes ont été accompagnées par le développement de traitements non chirurgicaux visant à corriger cette pathologie. A l'inverse, les recherches concernant l'éjaculation et ses anomalies n'ont pas connu le même développement.

Nous proposons une revue des données actuelles sur la physiologie de l'éjaculation afin d'envisager une approche physiopathologique des troubles de l'éjaculation et de rationaliser la réflexion médicale thérapeutique visant à les corriger. Les aspects pharmacologiques locaux et centraux ne seront pas abordés ici. Ils nous semblent en effet nécessiter 
une revue spécifique. L'éjaculation est une fonction complexe contrôlée par le système nerveux faisant intervenir des événements sécrétoires et musculaires. L'éjaculation nécessite une coordination des composantes sympathiques, parasympathiques et somatiques du système nerveux. Le contrôle de l'éjaculation fait par ailleurs intervenir différents étages du système nerveux central qui seront décrits.

L'éjaculation peut être définie comme une série d'événements aboutissant à l'expulsion du sperme au méat urétral. L'éjaculation est contemporaine de l'orgasme. Cependant on sait que l'éjaculation n'est pas synonyme d'orgasme. L'orgasme se rapporte en effet à une sensation de plaisir objective. Chez l'homme l'orgasme est une réponse physiologique complexe associant

1) éjaculation,

2) manifestations extragénitales : tachycardie, polypnée, sensation de chaleur, etc... et

3) sensation de plaisir. Dans des conditions expérimentales et pathologiques, éjaculation et orgasme peuvent être dissociés.

L'éjaculation comprend deux phases distinctes: l'émission et l'expulsion. Ces deux phases sont médiées par des réflexes spinaux polysynaptiques activés par les afférences honteuses internes (nerf pudendal), ces réflexes sont modulés par les centres supraspinaux. L'émission comprend la sécrétion du liquide séminal par les glandes sexuelles accessoires, la contraction du tractus séminal de l'épididyme à la prostate, assurant le transport les spermatozoïdes, et la fermeture du col vésical et du sphincter urétral externe. La phase d'expulsion fait intervenir des contractions rythmiques de la musculature lisse urétrale et de la musculature striée périnéale aboutissant à l'éjaculation du sperme proprement dite. La période réfractaire fait suite à l'éjaculation, durant celle-ci l'excitation sexuelle est inhibée.

\section{CONTRÔLE DE L'ÉJACULATION}

\section{a) Organisation anatomique de l'innerva- tion périphérique.}

L'innervation des structures impliquées dans la reproduction chez le mâle a été largement décrite $[11,32,39,72,148]$. L'ensemble des structures anatomiques impliquées dans la survenue de l'éjaculation reçoit à la fois une innervation sympathique et parasympathique. Celle-ci est essentiellement issue d'une part du plexus pelvien ou plexus hypogastrique inférieur qui représente un carrefour pour les fibres nerveuses des nerfs pelviens et hypogastriques et d'autre part de la chaîne sympathique paravertébrale lombo-sacrée. On considère généralement que l'émission est contrôlée exclusivement par le système nerveux sympathique, cependant une synergie entre l'innervation sympathique et parasympathique est la règle pour la plupart des fonctions viscérales. Ainsi des mécanismes adrénergiques et cholinergiques sont impliqués dans le contrôle nerveux de l'éjaculation ainsi que des mécanismes non adrénergiques non cholinergiques (NANC) représentés par des fibres peptidergiques, purinergiques et nitrergiques (monoxyde d'azote) récemment mises en évidence. L'innervation afférente du tractus séminal a fait l'objet de peu de travaux et son rôle fonctionnel demeure mal connu.

\section{Épididyme}

L'innervation de l'épididyme emprunte deux trajets : le plexus spermatique supérieur qui suit l'artère spermatique et le plexus spermatique inférieur issu du plexus pelvien qui suit le canal déférent [92]. Le plexus spermatique inférieur assure la majeure partie de l'innervation de l'épididyme. Chez la plupart des espèces animales étudiées, l'innervation du rete testis est assez pauvre, l'innervation est plus dense dans la tête de l'épididyme et s'enrichit encore dans la queue [32]. Cette distribution est retrouvée chez l'homme [8]. Des terminaisons adrénergiques et cholinergiques ont été mises en évidence au voisinage des fibres musculaires lisses épididymaires [56]. Les neuromédiateurs peptidergiques présents dans l'épididyme sont essentiellement le vasoactive intestinal polypeptide (VIP), le neuropeptide Y (NPY) et le calcitonin gene-related peptide (CGRP). Le NPY est colocalisé avec la noradrénaline dans les fibres nerveuses sympathiques [91]. Une colocalisation du VIP et de l'acétylcholine est possible mais n'a pas été 
démontrée. Les fibres nerveuses contenant du CGRP sont vraisemblablement des terminaisons de neurones sensitifs [91]. Des travaux ayant porté sur d'autres structures de l'appareil génito-urinaire, en particulier la vessie, ont démontré que les fibres nerveuses sensitives contenant du CGRP pouvaient exercer un rôle efférent en régulant la sensibilité d'autres fibres nerveuses sensitives et en exerçant un effet direct sur le muscle lisse [99].

\section{Canal déférent}

L'innervation et la pharmacologie du canal déférent ont été très largement étudiées. Le canal déférent représente en effet un modèle classique d'étude de la pharmacologie du système nerveux autonome. Le canal déférent reçoit une innervation dense adrénergique et cholinergique [39]. Ces deux composantes sont issues du plexus pelvien qui reçoit des fibres nerveuses préganglionnaires des nerfs hypogastriques et pelviens. Les vaisseaux du canal déférent reçoivent une innervation adrénergique, les corps cellulaires de ces neurones étant situés dans la chaîne sympathique caudale [153]. Parmi les fibres nerveuses adrénergiques et cholinergiques présentes dans le canal déférent, les fibres cholinergiques sont minoritaires, représentant environ 15 à $20 \%$ de l'ensemble des fibres. La plupart des fibres cholinergiques se concentrent à proximité de l'épithélium, cependant une innervation cholinergique de la musculaire interne a également été mise en évidence [179]. L'innervation adrénergique forme un riche plexus assurant l'innervation de l'ensemble des couches musculaires [91]. De très nombreux travaux ont décrit l'anatomie et la pharmacologie de l'innervation peptidergique du canal déférent. Le VIP et le NPY sont particulièrement abondants. NPY et noradrénaline sont colocalisés dans les terminaisons nerveuses sympathiques $[91,158]$. Il est probable, mais non démontré, que le VIP et l'acétylcholine soient également colocalisés. Des enképhalines, la substance $\mathrm{P}$ (SP), la somatostatine et le CGRP à ce niveau ont également été mis en évidence dans des terminaisons nerveuses [32]. Le CGRP et la SP sont vraisemblablement présents dans les terminaisons sensitives et pourraient jouer un rôle en modulant le contrôle efférent [98].

\section{Vésicules séminales}

Les vésicules séminales reçoivent une innervation sympathique et parasympathique issue des nerfs hypogastriques et pelviens via le plexus pelvien [153, 1, 39, 182]. L'innervation adrénergique se distribue dans les couches musculaires lisses interne et externe et est absente de l'épithélium. A l'inverse, l'innervation cholinergique se distribue dans l'épithélium et est très peu abondante dans les couches musculaires. Des fibres nerveuses VIPergiques ont une distribution comparable et le VIP est vraisemblablement colocalisé avec l'acétylcholine [168]. Le NPY est colocalisé avec la noradrénaline dans les terminaisons sympathiques [158].

\section{Prostate}

L'innervation et la pharmacologie prostatiques ont fait l'objet de nombreux travaux visant à la mise au point de traitements pharmacologiques ayant pour but de réduire les symptômes irritatifs et obstructifs liés à l'hypertrophie prostatique bénigne. L'existence d'une innervation sympathique et parasympathique issue des nerfs hypogastriques et pelviens a été démontrée. Il existe une innervation adrénergique dense de la musculature lisse [153, 166, 167]. Une innervation cholinergique moins dense a été retrouvée à la fois dans la musculature lisse et dans l'épithélium [40,54]. Des études fonctionnelles et pharmacologiques ont montré qu'une partie du contrôle cholinergique prostatique est médiée par des fibres sympathiques cholinergiques [156]. Certains auteurs ont mis en évidence un contrôle parasympathique cholinergique [38, 46] alors que d'autres ne l'ont pas retrouvé [176]. L'utilisation de techniques de marquage rétrograde transynaptique des voies nerveuses a démontré que, chez le rat, des neurones préganglionnaires sympathiques et parasympathiques projettent vers la prostate [106]. Le VIP est le principal neuromédiateur peptidergique présent dans la prostate [168]. Il est vraisemblablement colocalisé avec l'acétylcholine comme dans d'autres parties du tractus urogénital. L'innervation NPYergique est dense dans la prostate, le NPY est vraisemblablement colocalisé avec la noradrénaline [65]. Il existe une innervation prostatique afférente dont le rôle est inconnu (164]. 


\section{Col vésical et urètre}

L'innervation du col vésical et de l'urètre a fait l'objet d'une revue générale [101]. Il existe une double innervation sympathique et parasympathique. L'innervation sympathique aurait pour origine le nerf hypogastrique et la chaîne sympathique paravertébrale lombo-sacrée. Cette innervation sympathique est responsable du maintien de la fermeture du col vésical et de l'urètre proximal. La présence d'une innervation parasympathique cholinergique a également été démontrée [175]. La relaxation active du muscle lisse du col vésical et de l'urètre prostatique commandée par l'innervation parasympathique serait médiée par des neuromédiateurs NANC comme le VIP et le monoxyde d'azote [134]. Des fibres sensitives sont également présentes dans le col vésical et l'urètre, elles libèrent de la SP et du CGRP [2]. $\mathrm{Au}$ sein de l'épithélium urétral et des canaux prostatiques, il existe de nombreuses cellules paracrines. Ces cellules contiennent de la sérotonine et parfois des peptides comme la somatostatine [37]. En microscopie électronique, il a été démontré que ces cellules paracrines présentent des microvillosités dans leur lumière, ceci suggère un rôle hémosensitif. De nombreuses cellules paracrines présentent un prolongement s'étendant vers la couche sous épithéliale. Il a été démontré que les cellules paracrines urétrales pourraient jouer un rôle facilitateur dans la survenue des réflexes sexuels [114].

\section{Muscles périnéaux}

La musculature striée périnéale comprend les muscles ischiocaverneux, bulbospongieux et releveurs de l'anus qui sont innervés par le nerf honteux ou pudendal [147].

\section{b) Neurophysiologie et pharmacologie périphériques}

Il existe une synergie d'activité entre l'innervation sympathique et parasympathique dans la commande de l'émission. Celle-ci fait intervenir la sécrétion épithéliale et la contraction musculaire lisse de l'ensemble du tractus séminal. La neurotransmission noradrénergique semble être prédominante dans la survenue de l'émission, mais il existe d'autres acteurs neu- rochimiques. La contraction de la musculature lisse est étroitement dépendante du contrôle adrénergique, alors que la sécrétion épithéliale est médiée par l'innervation cholinergique et peptidergique. La stimulation des nerfs sympathiques provoquent une réponse contractile du canal déférent $[81,93,177]$. La stimulation du nerf pelvien provoque également une contraction du canal déférent [88]. La contraction induite par la stimulation sympathique est inhibée par un bloquant des récepteurs alpha 1 adrénergiques : la prazosine, et la contraction médiée par l'innervation parasympathique est, quant à elle, partiellement inhibée par l'atropine, antagoniste muscarinique [89]. Les contractions induites par la stimulation sympathique pourraient être aussi médiées par la libération de purines en particulier l'ATP par les terminaisons nerveuses noradrénergiques [117, 118].

L'hypothèse d'une stimulation cholinergique de la sécrétion épithéliale du canal déférent a été proposée [3]. Cependant le contrôle nerveux de la sécrétion du canal déférent demeure mal connu.

La contraction des vésicules séminales est provoquée par la stimulation des voies sympathiques [136]. Des agonistes adrénergiques et cholinergiques contractent la vésicule séminale $[47,163]$. Il est intéressant de souligner que les contractions des vésicules séminales provoquées par la stimulation électrique du nerf hypogastrique ne sont pas totalement abolies par des antagonistes adrénergiques ou cholinergiques. Ceci amène à faire l'hypothèse de l'existence d'un autre neuromédiateur impliqué, un bon candidat semble être ici également l'ATP $[126,157]$.

La commande nerveuse de la sécrétion des vésicules séminales est mal connue. Chez le lapin, les lésions de l'innervation sympathique n'altèrent pas la sécrétion des vésicules séminales [66]. L'existence d'une innervation cholinergique de l'épithélium de la vésicule séminale conduit à faire l'hypothèse d'un rôle de cette composante dans le contrôle de la sécrétion [1].

Le contrôle nerveux de la fonction prostatique a fait l'objet de nombreux travaux [136, 156]. La stimulation de l'innervation sympathique 
provoque l'issue de liquide prostatique dans l'urètre [136, 155]. La stimulation des nerfs pelviens ne provoque pas la sécrétion d'un volume significatif de liquide prostatique. Les expériences pharmacologiques ont démontré que la sécrétion prostatique était dépendante d'une stimulation à la fois adrénergique et cholinergique [39]. Il existe une synergie des mécanismes adrénergiques et cholinergiques : la sécrétion de l'épithélium prostatique est stimulée par des mécanismes cholinergiques (muscariniques) et la contraction de la musculature lisse prostatique est médiée par des mécanismes adrénergiques (récepteurs alpha1) [82, 174]. L'innervation sympathique et parasympathique de la prostate est par ailleurs impliquée dans la croissance et le développement de la glande prostatique [116, 174].

La contraction du col vésical est dépendante de l'innervation sympathique $[34,136]$. La fermeture du col vésical est également médiée par des récepteurs alpha 1 adrénergiques.

La stimulation sympathique chez l'homme $[136,137]$ entraîne une séquence d'événements stéréotypés aboutissant à l'émission : fermeture du col vésical, contraction des vésicules séminales, contraction de la prostate et des canaux déférents. Ces observations ont conduit à conclure que l'émission était dépendante de la seule innervation sympathique. Cependant ces études n'ont pas concerné le contrôle de la sécrétion par l'épithélium des glandes sexuelles accessoires. Les données anatomiques et pharmacologiques suggèrent qu'il existe ici un contrôle parasympathique. Ainsi une conclusion générale concernant le contrôle autonome périphérique de l'émission peut être proposée: les mécanismes parasympathiques cholinergiques participent au contrôle de la sécrétion épithéliale et les mécanismes sympathiques adrénergiques sont responsables de la contraction musculaire lisse.

\section{c) Contrôle spinal}

\section{Afférences}

Les afférences honteuses internes (pudendales) en particulier les afférences péniennes empruntant le nerf dorsal de la verge ont un rôle primordial dans la médiation des réflexes d'émission et d'éjaculation proprement dite. L'innervation sensitive des canaux déférents, de la prostate et de l'urètre a été décrite. Cependant il faut répéter que le rôle fonctionnel de celle-ci concernant l'éjaculation demeure inconnu. Les afférences pudendales se projettent aux niveaux médullaires lombaires bas et sacrés hauts. Les terminaisons sensitives sont situées dans la partie médiane de la corne dorsale et- la commissure grise dorsale. Les afférences pudendales projettent également dans les parties latérales de la corne dorsale $[11,139,165]$. Chez le rat des projections vers les motoneurones de la corne ventrale ont par ailleurs été décrites [128].

La majorité des afférences péniennes chez l'homme sont de faible calibre, faiblement myélinisées ou non myélinisées, il s'agit de fibres de catégorie A delta ou C [58]. Contrairement à une idée classique, la sensibilité tactile du pénis est faible comparativement à celle d'autres régions cutanées [62] bien que la densité des fibres sensitives péniennes soit la plus importante de l'ensemble du corps [74]. Les études anatomiques et physiologiques ont décrit les propriétés des afférences péniennes $[22,23,55,59,74,75,76,77,86]$. La majorité des afférences péniennes sont non encapsulées. Elles médieraient des réponses afférentes d'intensité faible, d'adaptation lente $[22,86]$ et des réponses à seuil de déclenchement élevé [77]. Le recrutement de ces fibres sensitives exerce une effet inhibiteur sur les contractions réflexes des muscles ischiocaverneux et bulbospongieux. Les terminaisons nerveuses non encapsulées situées dans le gland [55, 74] pourraient médier les réponses sensitives d'adaptation rapide aux stimulations du gland [73]. L'innervation sensitive du gland reçoit des fibres sympathiques [129], cette organisation pourrait présenter le support pour un contrôle efférent de la sensibilité pénienne durant l'érection [76]. La sensibilité des récepteurs péniens augmente durant l'érection [79]. Les modifications de la compliance tissulaire, la modulation efférente par l'innervation sympathique et l'augmentation de température pendant l'érection pourraient expliquer cette variation de sensibilité [75]. Les modifications des mécanismes de la sensibilité pénienne 
durant l'érection observées chez l'animal doivent être extrapolées à l'homme avec précaution, les études humaines n'ayant été réalisées qu'à l'état flaccide. De nombreuses questions demeurent donc, relatives aux mécanismes de régulation de la sensibilité impliquée dans la survenue de l'érection, de l'émission et de l'éjaculation proprement dite.

\section{Efférences}

Les neurones sympathiques préganglionnaires à destinée pelvienne sont situés dans la colonne intermédiolatérale et la commissure grise dorsale de la moelle thoracique basse et lombaire haute $[60,120,124]$. Les neurones préganglionnaires situés dans la colonne intermédiolatérale projettent préférentiellement vers les cellules ganglionnaires de la chaîne sympathique paravertébrale lombaire et participent vraisemblablement au contrôle cardio-vasculaire. A l'inverse les neurones situés dans la commissure grise dorsale projettent préférentiellement vers les ganglions prévertébraux et sont principalement impliqués dans le contrôle de la motricité viscérale [72]. L'organisation spécifique des neurones issus de ces noyaux spinaux intervenant dans le contrôle de l'émission n'est pas connue.

Les neurones parasympathiques préganglionnaires sont situés dans la colonne intermédiolatérale des segments sacrés, regroupés dans le noyau parasympathique sacré $[61,121,121$, $122,123,125]$. Ce noyau renferme des neurones qui contrôlent la motricité de l'ensemble des viscères pelviens. Une somatotopie des neurones destinés à la vessie et au côlon a été mise en évidence chez le chat [35]. Les neurones préganglionnaires innervant la prostate ont été mis en évidence à l'aide d'une technique permettant le marquage rétrograde transsynaptique utilisant un virus neurotrope chez le rat, mais il n'existe pas de somatotopie dans cette espèce [105].

Les motoneurones pudendaux innervant la musculature striée périnéale sont situés dans le noyau d'Onuf de la moelle lombo-sacrée [90, $148,165,166]$. Chez le rongeur, les motoneurones pudendaux sont divisés en deux noyaux, l'un contenant les neurones innervant le muscle bulbospsongieux et le sphincter anal externe et le second le muscle ischiocaverneux et le sphincter urétral externe [111, 165].

\section{Interneurones}

Les interneurones spinaux et les réseaux neuronaux contrôlant tous les réflexes sexuels connus sont polysynaptiques. Cela signifie que des interneurones sont nécessaires pour transformer à l'étage spinal des informations sensitives en signaux moteurs ou sécrétoires. Ainsi la neurophysiologie et la neurochimie spinales sont déterminantes dans l'expression des réponses sexuelles. La connaissance des réseaux d'interneurones spinaux impliqués dans le contrôle des fonctions sexuelles est à ce jour très incomplète. Les seules données acquises l'ont été chez l'animal. De plus les caractéristiques spécifiques des neurones impliqués dans le contrôle de l'érection, de l'émission ou de l'éjaculation sont inconnues rendant inaccessible la description du système en réseau qui aboutit à partir d'une stimulation sexuelle à la série d'événements moteurs coordonnés aux étages thoraco-lombaire et sacré qui constituent l'éjaculation.

Les neurones qui projettent sur les motoneurones honteux ou sur les neurones préganglionnaires autonomes ont été mis en évidence à l'aide d'expériences de transport transsynaptique rétrograde utilisant l'agglutinine de germe de blé (wheat germ agglutinin, WGA) ou des virus neurotropes. Ces molécules complexes sont transportées par le flux axonal rétrograde du point où elles ont été injectées vers les somas des neurones (neurones de premier ordre) puis franchissent les synapses et gagnent ainsi les neurones de second ordre qui projettent sur les précédents. L'injection unilatérale de WGA dans le muscle bulbospongieux révèle ainsi des interneurones putatifs bilatéralement dans la corne ventrale et la substance grise intermédiaire, cette dernière comprenant la commissure grise dorsale [28, 131]. Des résultats comparables ont été obtenus après l'injection du virus pseudorage [105] ou de virus rabique [162] dans les muscles striés périnéaux. L'injection de virus pseudorage dans les corps caverneux, l'urètre ou la vessie $[107,170]$ révèle également des neurones immunoréactifs pour le virus dans la commissure grise et l'aire $\mathrm{X}$ autour du canal central. 
En accord avec ces données, la présence de neurones immunoréactifs pour la protéine Fos dans la substance grise a été objectivée après la mise en jeu des réflexes sexuels ou la stimulation $\mathrm{du}$ nerf dorsal $\mathrm{du}$ pénis [133]. L'identification des interneurones de la moelle épinière est particulièrement importante, puisqu'une grande partie des contrôles exercés par les structures supraspinales sur les réponses sexuelles s'applique sur ces interneurones. L'étude des interneurones spinaux mis en jeu dans les fonctions sexuelles est aujourd'hui peu développée. Chez le chat anesthésié, la stimulation des afférences honteuses provoque des potentiels de champ dans les couches $\mathrm{V}$ et VI des segments spinaux L7 à S2 [48]. La partie médiane de la corne dorsale, la commissure grise dorsale et l'aire X contiennent des neurones répondant à la stimulation des viscères pelviens et du périnée $[36,66,69$, $70,77,78]$. Une caractéristique commune à toutes ces études, en accord avec les résultats des études utilisant le transport axonal rétrograde transsynaptique et la révélation de la protéine Fos, est la mise en évidence systématique de neurones dans la partie centrale de la moelle épinière. Ceci suggère fortement la contribution de ces neurones au contrôle spinal des fonctions pelviennes. Cependant de nombreuses études sont encore nécessaires avant que l'on puisse dégager des concepts clairs quant à leur rôle dans la commande spinale de l'éjaculation.

\section{Réflexes segmentaires}

- Réflexe bulbocaverneux:

Le réflexe bulbocaverneux est un réflexe spinal segmentaire. Il est mis en jeu par la pression légère du pénis et consiste en la contraction des muscles périnéaux $[16,41,135,171]$. La volée afférente du réflexe est représentée par la branche sensitive du nerf honteux et la volée efférente par les axones des motoneurones honteux. Chez le rat, ce réflexe est polysynaptique [112]. Le rôle physiologique du réflexe bulbocaverneux n'est pas clairement établi. Cependant, durant l'excitation sexuelle, la contraction des muscles bulbospongieux et ischiocaverneux provoquerait ou augmenterait la rigidité pénienne $[94,95,96,146]$. La contraction du sphincter urétral externe pour- rait faciliter l'accumulation de fluide séminal dans l'urètre postérieur [101].

- Fermeture du col vésical:

La contraction du col de la vessie est contrôlée principalement par l'innervation sympathique. La stimulation des afférences honteuses inhibe l'activité vésicale et ferme le col de la vessie.

\section{Emission:}

Les réflexes sous-tendant l'émission séminale n'ont pas été beaucoup étudiés. La stimulation des afférences péniennes provoquait l'émission séminale et la contraction du canal déférent, en réponse à la mise en jeu combinée des efférences sympathiques et parasympathiques [88]. Chez le lapin et chez le rat, des contractions des vésicules séminales sont enregistrées 100 à $300 \mathrm{~ms}$ après l'intromission, ce qui suggère qu'elles sont provoquées par la stimulation du pénis [13, 30].

\section{Ejaculation:}

La phase finale de l'éjaculation est représentée par la contraction rythmique des muscles striés périnéaux et des muscles lisses de l'urètre $[12,15 ; 50 ; 132]$. Cette réponse est caractérisée par l'activité synchrone des muscles ischiocaverneux, bulbospongieux, des sphincters anal et urétral externes, et du releveur de l'anus. Les contractions sont remarquablement régulières. L'intervalle entre les bouffées d'activité électromyographique est de 0,6 seconde, puis augmente d'un pas de $100 \mathrm{~ms}$ à chaque intervalle suivant [15]. La réponse complète chez l'homme comprend 10 à 15 contractions. L'organisation des bouffées d'activité électrique est relativement invariante chez un même sujet. Alors que l'activité des muscles striés est une caractéristique remarquable, l'activité coordonnée des voies nerveuses sympathique, parasympathique et somatique a été mise en évidence pendant cette réponse chez l'animal [113].

Il faut signaler que les contractions des muscles striés périnéaux enregistrées chez l'homme et la femme au cours de l'orgasme sont exactement comparables [14, 15]. Ces données, auxquelles s'ajoutent les identités entre les expériences subjectives rapportées par les sujets et les réponses extragénitales, 
ont conduit à émettre l'hypothèse de réseaux nerveux semblables à l'origine de l'orgasme dans les deux sexes $[85,109]$. Une base expérimentale confirmant cette hypothèse a été fournie chez le rat [114]. La compréhension de ces mécanismes doit passer par une comparaison et la recherche des identités et des différences fonctionnelles dans les deux sexes.

Le mécanisme sensoriel qui déclenche l'ensemble de la séquence motrice de l'éjaculation demeure inconnu. Une théorie propose la stimulation de l'urètre par l'accumulation de fluide séminal. Chez l'animal, la distension de l'urètre avec une solution salée [113] ou par l'émission séminale [83] provoque de telles réponses. Cependant, quoique ces stimulations soient capables de provoquer l'éjaculation, des données expérimentales et cliniques ne confirment pas cette hypothèse. L'anesthésie de l'urètre ou l'inhibition de la sécrétion séminale par la guanéthidine n'empêche pas l'éjaculation chez le rat pendant la copulation [67]. Une activité motrice identique à celle développée pendant l'éjaculation a été mise en évidence chez des patients après cystoprostatectomie comprenant évidemment l'exérèse des vésicules séminales [12] et chez des patients sains volontaires après inhibition de l'émission séminale par la phénoxybenzamine [50].

On reconnaît volontiers aujourd'hui un rôle prépondérant aux fibres nerveuses sensitives du nerf honteux dans la transmission des informations à l'origine de l'éjaculation. Cependant la nature exacte des terminaisons nerveuses périphériques impliquées et leur localisation demeurent inconnues. La stimulation des afférences honteuses chez l'animal provoque des réponses réflexes motrices de type éjaculatoire $[113,130]$. L'anesthésie du gland augmente la latence d'éjaculation chez l'animal. Cette observation a permis de proposer cette modalité thérapeutique pour traiter des patients souffrant d'éjaculation prématurée. On a besoin aujourd'hui de mieux définir la nature des afférences péniennes impliquées pour aborder ce trouble.

L'éjaculation est un mécanisme spinal, qui persiste après section médullaire $[17,21,113$, 130]. La difficulté d'obtenir une éjaculation après traumatisme médullaire est largement documentée [20]. Cependant, lorsque les segments lombo-sacrés de la moelle épinière sont épargnés par la lésion, une éjaculation peut être provoquée chez des patients à section spinale complète [21]. Ces données montrent que le système moteur à l'origine des contractions stérotypées des muscles striés périnéaux se trouve dans la moelle épinière. De plus l'émission réflexe, la fermeture du col vésical et le réflexe bulbocaverneux sont tous des réflexes spinaux très probablement provoqués par le recrutement des afférences sensitives du nerf honteux. Ainsi, il est très vraisemblable que tous les acteurs nerveux de l'éjaculation sont présents dans la moelle épinière lombo-sacrée. Ce fait a été exploité avec succès pour recueillir du sperme chez des patients traumatisés médullaires en réponse à une stimulation de type vibratoire appliquée sur le pénis. Les centres supraspinaux facilitent ou inhibent l'éjaculation, adaptant ainsi le réflexe sexuel au contexte.

\section{d) Contrôle supraspinal de l'éjaculation:}

Il y a 30 ans, F. Beach, dans une revue décrivant le contrôle nerveux et endocrinien du comportement sexuel, expliquait que les réponses sexuelles recrutant les organes génitaux pouvaient être considérées comme autant de réflexes placés sous un contrôle supraspinal. En outre, le contrôle hormonal du comportement sexuel pouvait expliquer en grande partie par la modulation par les hormones de l'activité des centres supérieurs [9]. Ces propositions sont toujours valides, à la lumière de données expérimentales plus récentes [140, 141].

On doit insister sur le fait qu'aujourd'hui notre connaissance des mécanismes nerveux centraux intervenant dans le contrôle les fonctions génitales et le comportement sexuel sont très incomplètes. La plupart des données disponibles est issue de travaux menés chez l'animal, et l'analogie avec les mécanismes présents chez l'homme doit être discutée. Il y a par exemple de bonnes raisons expérimentales pour penser que l'olfaction joue un rôle majeur dans le comportement sexuel des rongeurs. Il est douteux qu'un tel mécanisme intervienne d'une façon aussi importante chez l'homme 
quoiqu'en pensent les fabricants de parfum...Un problème majeur réside dans l'identification des structures nerveuses qui contrôlent différents apects du comportement sexuel chez le mâle tels que la motivation, l'orientation et l'appétence sexuelles, l'érection et l'éjaculation.

L'aire préoptique médiane de l'hypothalamus (medial preoptic area : MPOA) a fait l'objet d'un grand nombre de recherches dans le cadre du contrôle central du comportement sexuel chez le mâle. MPOA est un noyau clé dans la régulation des fonctions sexuelles mâles et représente le site essentiel de l'action des androgènes sur le comportement sexuel. Des lésions de MPOA causent de profondes altérations du comportement sexuel chez le rat mâle, éliminant toute tentative de monte [63]. Depuis ces observations princeps, des résultats comparables y compris chez les primates ont été observés chez un grand nombre de vertébrés, mais également chez les poissons et les reptiles[154]. MPOA contient un grand nombre de neurones qui possèdent des récepteurs des hormones stéroïdes [144] et des implants de testostérone dans MPOA chez le rat castré restaurent l'activité sexuelle [33].

La stimulation électrique ou chimique de MPOA active le comportement et les fonctions sexuelles chez l'animal éveillé [33, 71, 100, 119] et provoque érection et éjaculation chez le rat anesthésié $[51,103]$. Cette stimulation réduit le nombre d'intromissions avant l'éjaculation, réduit l'intervalle entre les intromissions et réduit la période réfractaire post-éjaculatoire. L'évolution de ces paramètres met en évidence un effet facilitateur de MPOA sur la performance sexuelle. De plus, la stimulation de MPOA peut provoquer une émission séminale et une éjaculation chez le rat [64, 169] et chez le singe [138] sans excercer de stimulation génitale. Il reste cependant à établir si des fonctions sexuelles différentes comme l'érection et l'éjaculation sont contrôlées différemment au sein de MPOA.

Il est également nécessaire de préciser si les stimulations de MPOA affectent la motivation sexuelle, la performance ou les deux. Des résultats expérimentaux suggèrent que les lésions de MPOA empêchent l'animal d'initiali- ser le comportement sexuel, sans pour autant altérer les capacités d'érection ou d'éjaculation ni abaisser la motivation sexuelle. Everitt et Stacey [43] ont étudié la motivation sexuelle en utilisant le paradigme du renforcement de deuxième ordre, expérience au cours de laquelle des rats mâles ont accès à des femelles réceptives en appuyant sur une pédale. Chez ces animaux, les lésions de MPOA entraînent des inhibitions de la copulation, mais ne réduisent pas le nombre d'appuis réalisés sur la pédale. Chez le singe, la lésion de MPOA supprime monte, intromission et éjaculation en présence d'une femelle en œstrus [154]. Cependant les mâles continuent à appuyer sur une pédale pour accéder à ces femelles et se masturbent toujours jusqu'à l'éjaculation. On conclut ainsi en insistant sur le fait que la lésion de MPOA peut abolir le comportement sexuel en interrompant la capacité du mâle à se lancer dans des interactions socio-sexuelles appropriées.

De nombreuses autres questions au sujet du rôle de MPOA demeurent encore sans réponse. En particulier on ignore la nature des voies nerveuses par lesquelles MPOA contrôle le réseau spinal des réflexes sexuels. MPOA, en effet, n'émet pas de projections directes vers la moelle épinière [151]. Il y a donc des relais dans l'hypothalamus ou dans le tronc cérébral qui sont les intermédiaires de la facilitation exercée par MPOA sur les fonctions sexuelles. Rechercher ces structures relais est très difficile du fait de la complexité de MPOA. MPOA intervient dans un grand nombre d'activités complexes comme le comportement parental, la soif et la prise hydrique, la régulation de la température, et des fonctions neuroendocrines. Cette complexité fonctionnelle est sous-tendue par les relations multiples entre MPOA et de larges ensembles du système limbique et du tronc cérébral [151, 152].

\section{L'amygdale}

L'amygdale est une autre structure nerveuse jouant un rôle important dans le contrôle du comportement sexuel. Certains neurones de l'amygdale (principalement dans sa partie médiane) expriment le gène d'expression c-fos, c'est-à-dire sont activés, pendant la copulation [7, 31]. Kluver et Bucy [87] ont décrit l'hyper- 
activité sexuelle chez le singe après des lésions larges du lobe temporal, incluant l'amygdale. En revanche des lésions plus limitées de l'amygdale n'induisent pas un comportement d'hypersexualité, mais plutôt une hypoactivité sexuelle. Les lésions de la partie basolatérale de l'amygdale interrompent la recherche par le mâle d'une partenaire, alors que sa capacité à copuler demeure intacte [44]. On en conclut que l'amygdale joue un rôle important dans l'aspect motivationnel du comportement sexuel mais pas dans son aspect de performance. A cet égard, l'amygdale peut jouer un rôle complémentaire de MPOA. Les deux structures entretiennent des relations réciproques [151, 152] et ensemble représentent des éléments essentiels du contrôle du comportement sexuel par le système nerveux central. Il reste à explorer le rôle plus précis de l'amygdale et de son noyau médian dans les mécanismes de l'érection et de l'éjaculation.

\section{Le noyau paraventriculaire}

Le noyau paraventriculaire de l'hypothalamus (paraventricular nucleus, PVN) a également fait l'objet d'études démontrant son rôle dans le contrôle des fonctions sexuelles. PVN est une structure clé de l'intégration neuroendocrine et autonome [161]. Les neurones parvocellulaires du PVN émettent des axones qui gagnent la moelle épinière [143] et les motoneurones honteux [172]. Les projections vers la moelle lombo-sacrée sont principalement ocytocinergiques, et les neurones parvocellulaires ocytocinergiques projettent préférentiellement vers la moelle lombo-sacrée. Les concentrations d'ocytocine circulante et dans le liquide céphalo-rachidien augmentent pendant l'excitation sexuelle [24] et après l'éjaculation et l'orgasme. PVN reçoit des projections denses de MPOA [151]. Les neurones du PVN sont activés lorsqu'on stimule les fibres sensitives péniennes [180]. La stimulation du PVN augmente la pression intracaverneuse chez le rat anesthésié [25] et provoque l'éjaculation [115]. Certains neurones du PVN sont immunoréactifs pour le virus de la pseudorage ou de la rage après que ceux-ci aient été injectés dans les corps caverneux [107] ou les muscles ischiocaverneux et bulbospongieux [105, 162]. L'ensemble de ces données permet d'affirmer le rôle de PVN dans le contrôle central des fonctions sexuelles.

\section{Tronc cérébral}

Deux études ont montré une augmentation importante de la période réfractaire post-éjaculatoire après des lésions de la partie rostroventrale du tronc cérébral $[6,27]$. Les auteurs ont expliqué ces effets par la lésion de systèmes de projections ascendantes monoaminergiques. Cependant dans ces études, les lésions réalisées étaient étendues et d'autres structures étaient également lésées. Il n'y a pas eu depuis des recherches plus précises dans ce domaine. Chez le rat, la lésion de l'aire tegmentale ventrale provoque des altérations du comportement sexuel [18, 19]. L'aire tegmentale ventrale envoie des projections vers et reçoit des projections de MPOA [151, 152]. L'aire tegmentale ventrale serait principalement impliquée dans le relais des informations génitales vers le diencéphale [7, 149].

La substance grise périaqueducale entretient également des relations réciproques avec MPOA. Après lésion de la substance grise périaqueducale, on mesure une accélération de la copulation chez le rat [19]. Ces lésions interrompent des faisceaux de voies nerveuses ascendantes et descendantes. Dans l'ensemble, si l'on peut affirmer un rôle des structures du tronc cérébral dans le contrôle des fonctions sexuelles, de nombreux détails de ce contrôle sont aujourd'hui inconnus.

\section{Noyau paragigantocellulaire}

Une des conclusions formulées par Beach [9] au sujet du contrôle par le système nerveux central des fonctions sexuelles était que les réflexes sexuels médiés à l'étage spinal étaient inhibés par des structures supraspinales. Une telle structure a récemment été identifiée chez le rat [104]. Une petite population de neurones dans la partie rostrale du noyau paragigantocellulaire ( $\mathrm{nPGi}$ ) est responsable d'une forte influence inhibitrice s'exerçant sur l'éjaculation réflexe. Ces neurones projettent sur les neurones préganglionnaires autonomes et les interneurones de la moelle lombo-sacrée [108]. Ils sont immunoréactifs pour des virus neurotropes injectés dans les corps caverneux ou les muscles striés périnéaux [105, 107, 162]. La 
lésion électrolytique ou chimique de nPGi facilite les érections réflexes [108] et le comportement sexuel [181]. La plupart des neurones du nPGi projetant vers la moelle est sérotoninergique [107]. Ces données fournissent un support neurochimique aux effets des composés qui interfèrent avec l'éjaculation, tels que la clomipramine, qui augmente les concentrations de sérotonine.

\section{DYSÉJACULATIONS}

Les dyséjaculations ne sont habituellement pas considérées comme une entité clinique unique, malgré l'altération de mécanismes physiologiques communs. Par exemple, il est logique de postuler que la régulation descendante cérébro-spinale du réflexe médullaire contrôlant les mécanismes locaux de l'éjaculation (cf supra) est altérée inversement lorsqu'il existe une éjaculation prématurée ou une éjaculation retardée. Il est intéressant de remarquer que jusqu'à un passé récent les urologues étaient essentiellement impliqués dans les dyséjaculations responsables d'infertilité, c'est à dire l'éjaculation rétrograde et l'anéjaculation alors que les dyséjaculations entrant dans le cadre des dysfonction sexuelles sans infertilité, c'est à dire l'éjaculation prématurée ou retardée étaient essentiellement prises en charge par les sexothérapeutes.

\section{a) Ejaculation prématurée}

L'éjaculation prématurée est la dysfonction sexuelle la plus fréquente chez les hommes adultes [4]. Sa prévalence dans la population masculine adulte a été estimée à $30 \%[4,10$, 49]. Il n'existe pas de consensus pour une définition. Il y a autant de définitions que d'auteurs ayant écrit sur l'éjaculation prématurée... Une définition validée est particulièrement souhaitable afin de choisir un qualificatif : rapide ou prématurée et surtout de proposer un cadre bien défini comme cela a été réalisé pour la dysfonction érectile [127]. Il s'agit d'une condition importante à la réalisation d'essais cliniques visant à évaluer l'efficacité de médicaments dans le traitement de ce trouble. Brièvement les définitions qui ont été proposées font référence à :
1. l'idée de satisfaction de la partenaire et parfois du patient $[53,110]$.

2. l'incapacité à contrôler de façon consciente le moment de l'éjaculation, s'appuyant sur le contrôle volontaire de ce qui a été appelé "le réflexe éjaculatoire" [79].

3. nombre d'allers et retours intra-vaginaux avant l'éjaculation ou le délai écoulé entre la pénétration vaginale et l'éjaculation [84].

4. l'éjaculation ante-portas définie comme survenant avant la pénétration vaginale ou sans qu'une érection rigide se soit développée [145], ici est introduite la notion de sévérité.

5. une combinaison de ces différents aspects en définissant l'éjaculation prématurée comme : "une éjaculation survenant de façon persistante ou récurrente à la suite d'une stimulation sexuelle minime, au moment ou un peu avant la pénétration et avant que l'homme le souhaite [5]".

Quelle que soit la définition utilisée, la demande du patient et/ou de sa partenaire peut être résumée au fait que lorsque le patient est stimulé sexuellement l'éjaculation survient (trop) rapidement.

Que savons-nous de la physiopathologie de l'éjaculation prématurée ? On a considéré dans le passé que l'éjaculation prématurée était due à des anomalies du veru montanum [57], dans ces circonstances la coagulation du veru a été réalisée [52]. Puis l'éjaculation prématurée a été considérée comme acquise et d'origine psychologique [4]. Une théorie somatique a également été avancée. Considérant l'incidence très élevée de l'éjaculation prématurée, très peu d'études cliniques ont été réalisées pour confirmer cette théorie [52] et l'examen attentif des concepts qui sous tendent cette théorie somatique conclut à leur caractère assez confus. Il est ainsi proposé que l'éjaculation prématurée est due à l'existence d'une hypersensibilité de la verge à la stimulation et/ou à une diminution du seuil de l'éjaculation. Il n'a pas été fait de distinction claire entre ces deux hypothèses. Quelques travaux bien conduits ont tenté de déterminer les seuils de sensibilité cutanée pénienne à la stimulation locale [141]. Mais ces 
seuils, ou les seuils de perception vibratiles du pénis [178], seraient différents des seuils de sensibilité des fibres afférentes recrutées lors de l'éjaculation. Le neurophysiologie de ces fibres demeure très mal connue (cf supra). De plus l'existence d'une différence de sensibilité pénienne entre des patients se plaignant d'éjaculation prématurée et des hommes de même âge ne rapportant pas ce trouble n'est pas établie [141, 178]. Une augmentation de l'amplitude sans modification de la latence du réflexe bulbo-caverneux a été retrouvée chez des patients présentant une éjaculation prématurée. Ceci a été interprété comme le signe d'une hyperexcitabilité réflexe ou d'une "altération de la modulation centrale" des motoneurones honteux internes ou pudendaux [29]. L'étude des potentiels évoqués corticaux en réponse à la stimulation du gland et/ou du nerf dorsal de la verge chez des patients se plaignant d'éjaculation prématurée a mis en évidence une plus grande amplitude de la réponse ainsi qu'une variation intermittente de sa latence. Il a été proposé que ces patients présentaient une augmentation de la représentation corticale des stimuli sensitifs de la verge responsable d'une hypersensibilité du gland [45, 178]. Cependant il n'est pas certain que l'ensemble de ces mesures explorent spécifiquement les voies nerveuses afférentes impliquées dans le réflexe d'éjaculation. De plus les voies afférentes autonomes motrices sympathiques, en particulier commandant l'éjaculation, ne sont pas accessibles à des explorations neurophysiologiques.

D'autres auteurs ont proposé d'intégrer l'éjaculation prématurée dans un approche beaucoup plus large bio-psychosociale incluant des éléments somatiques et comminutifs comme l'anxiété sexuelle ainsi qu'une perception altérée de l'excitation sexuelle [141]. Une diminution moindre des potentiels cutanés sympathiques durant des érections pharmacologiquement induites chez des patients se plaignant d'éjaculation prématurée par rapport à patients témoins pourrait témoigner d'une anxiété sexuelle participant aux mécanismes de l'éjaculation prématurée [42]. Cependant des approches psychophysiologiques tendent à conclure que l'anxiété ne serait pas un élément étiologique obligatoire de l'éjaculation préma- turée [142,160]. Le rôle possible d'une fréquence abaissée des rapports ou de la masturbation dans la pathogénie de l'éjaculation prématurée a également été proposé mais n'a pas été confirmé [159].

En conclusion il semble que l'éjaculation prématurée pourrait être le fait d'une hypersensibilité à la stimulation sexuelle périphérique et centrale, mais la démonstration physiopathologique d'une telle perturbation n'a pas été formellement fournie. Il pourrait être intéressant de s'interroger sur le rôle de la moelle épinière siège de la modulation à la fois des afférences génitales et des efférences motrices commandant les mécanismes de l'éjaculation. La survenue d'émission prématurée dans les suites de traumatismes de la moelle épinière au niveau D12 L1 supporte cette hypothèse [80]. Dans cette étude, il a été proposé que chez ces patients blessés médullaires les voies nerveuses descendantes empruntant le faisceau spino-thalamique pourraient être intactes alors qu'existerait une perte de l'inhibition d'origine supra spinale.

Les différences entre l'éjaculation prématurée primaire et secondaire ont également été soulignées, se basant sur des mesures du réflexe bulbo-caverneux [52]. De plus l'éjaculation prématurée secondaire est habituellement considérée comme consécutive ou associée à l'insuffisance érectile alors qu'à l'inverse l'insuffisance érectile peut compliquer une éjaculation prématurée primaire.

\section{b) Les autres troubles de l'éjaculation}

L'incidence des troubles de l'éjaculation à l'exception de l'éjaculation prématurée, est très mal connue. Des difficultés à éjaculer ont été rapportées par $4 \%$ d'hommes adultes $[49,10]$. Nous proposons de considérer l'éjaculation retardée avec l'anéjaculation qui comprend l'éjaculation rétrograde et le défaut d'émission, la différence entre ces deux dernières dysfonctions étant le plus souvent ignorée. L'ensemble de ces dysfonctions partage en effet des caractéristiques physiopathologiques communes.

L'éjaculation retardée a été rapportée chez $4 \%$ d'hommes adultes présentant une dysfonction sexuelle $[10,110]$. Elle peut être iatrogène pro- 
voquée par les mêmes substances pharmacologiques que celles responsables de l'absence d'émission [cf infra] et a des facteurs psychologiques inhibiteurs.

L'absence d'éjaculation antégrade due à une éjaculation rétrograde totale est caractérisée par la persistance de l'émission sans éjaculation proprement dite, c'est à dire sans expulsion tonique du sperme au méat urétral. Le sperme est recueilli dans la vessie avec la spermaturie pathognomique lors de la miction après masturbation.

\section{L'éjaculation rétrograde est souvent iatrogène :}

1- par lésion incomplète de l'innervation périphérique sympathique lors de la chirurgie d'un anévrysme de l'aorte abdominale avec reconstruction aorto-iliaque, le curage ganglionnaire rétro péritonéal lombo-aortique pour les tumeurs testiculaires non séminomateuses, de l'exérèse du rectum pour des tumeurs malignes ou bénignes de la promonto-fixation $\mathrm{du}$ rectum dans le traitement des prolapsus rectaux (technique d'Orr-Loygues).

2- par altération de la musculature lisse responsable de la fermeture du col vésical : incision cervicale, résection transurétrale de prostate et adénomectomie prostatique rétropubienne pour hypertrophie bénigne de la prostate, plastie $\mathrm{Y}-\mathrm{V}$ du col vésical...

L'éjaculation rétrograde peut également être la conséquence d'une altération de la commande nerveuse contrôlant la fermeture du col vésical : neuropathie végétative périphérique du diabète, autres neuropathies systémiques en particulier maladie de Parkinson, sclérose en plaques...

L'éjaculation rétrograde peut également exister chez les patients blessés médullaires liée à une lésion du centre sympathique à l'étage thoraco lombaire.

Absence d'émission. Il s'agit d'un trouble très souvent iatrogène.

L'absence d'émission peut être la conséquence d'une lésion complète de l'innervation sympathique périphérique consécutive aux mêmes procédures chirurgicales que celles responsables d'éjaculation rétrograde.
La prostatectomie radicale ou la cystoprostatectomie sont évidemment du fait de l'exérèse prostatique responsables d'une disparition de l'émission.

En ce qui concerne la iatrogénie médicamenteuse, les mêmes drogues peuvent être responsables d'éjaculation retardée ou d'absence d'émission faisant intervenir des mécanismes proches. Deux catégories de substances pharmacologiques peuvent être définies. La première comprend de nombreuses molécules avec lesquelles ont été rapporté anecdotiquement une éjaculation retardée ou une anéjaculation. Un ouvrage récent à colligé l'ensemble de ces "case reports" et les données de pharmacovigilance à ce sujet. On retrouve le plus fréquemment des psychotropes, à ce propos il faut considérer que les effets secondaires concernant l'éjaculation de ces substances sont très largement sous déclarés par les patients compte tenu de leur difficulté à évoquer ces problèmes d'éjaculation avec leur thérapeute. Par ailleurs, la variation inter individuelle de la sensibilité à ces substances pharmacologiques devrait susciter plus d'intérêt... Il faut retenir que les altérations de l'éjaculation pharmacologiquement induites sont réversibles avec l'arrêt de la drogue responsable. Le second groupe de médicaments comprend des substances pour lesquelles le taux d'éjaculation retardée voire d'anéjaculation est si élevé quelles ont été utilisées dans des essais cliniques ayant pour but de traiter l'éjaculation prématurée. Ce groupe comprend des bloquants des récepteurs alpha adrénergiques, la clomipramine, la paroxetine ainsi que le groupe des inhibiteurs sélectifs de la recapture de la sérotonine.

Les neuropathies végétatives périphériques peuvent être responsables également d'éjaculation rétrograde, il faut citer ici en premier lieu le diabète.

Les traumatismes médullaires sont également volontiers responsables d'absence d'émission en fonction du niveau et du caractère complet au nom de la lésion. Il est remarquable que malgré la prévalence très élevée des troubles de l'éjaculation chez les patients blessés médullaires et diabétiques, les pourcentages respectifs d'éjaculation rétrograde et d'absence d'émission ne sont pas connus. 
L'hypospermie caractérisée par une diminution du volume de l'éjaculat peut être due à une obstruction des canaux éjaculateurs. Celle-ci est rarement congénitale associée avec des formes sévères d'hypospadias ou consécutif à des formes complexes d'épispasdias associé à une exstrophie vésicale. Les hypospermies acquises peuvent être dues à des prostatites ou des traumatismes urétraux instrumentaux et des sténoses urétrales. La diminution du volume de l'éjaculat peut être également la conséquence d'une éjaculation rétrograde partielle. Cependant la circonstance favorisante la plus fréquente de l'hypospermie est la diminution du volume de l'éjaculat liée à l'âge. Celle-ci demeure cependant peu documentée.

\section{CONCLUSION - PERSPECTIVES}

Si le mécanismes locaux de l'éjaculation ainsi que leur contrôle par le système nerveux périphérique efférent sont désormais mieux connus, les mécanismes à l'origine de la survenue de l'émission et de l'éjaculation proprement dite demeurent inexplorés. S'agit-il d'une stimulation chimique et/ou physique de la muqueuse urétrale associée à une stimulation du gland ? Quels sont les afférences qui sont recrutées, les neuromédiateurs libérés et les récepteurs médiant leur action?

Il est vraisemblable, qu'à l'exception de l'éjaculation rétrograde due à une lésion de la musculature lisse du col vésical, la quasi totalité des troubles de l'éjaculation, qu'il s'agisse d'éjaculation survenant trop rapidement, ou à l'inverse retardée ou d'absence d'éjaculation, trouve son origine pour partie ou en totalité au sein du système nerveux central. Ceci rend nécessaire un important effort de recherche visant à préciser les mécanismes tant spinaux que supraspinaux intervenant dans la commande de l'éjaculation. Seule cette connaissance permettra une approche pharmacologique adaptée visant à traiter l'ensemble de la pathologie de l'éjaculation.

\section{REFERENCES}

1. AL-ZUHAIR, A., GOSLING, J.A. DIXON, J.S.: Observations on the structure and autonomic innervation of the guinea-pig seminal vesicle and ductus deferens. J. Anat., 1975, 120: 81-93.
2. ALM, P., ALUMETS, J., BRODIN, E., HÅKANSON, R., NILSSON, G., SJÖBERG, N.-O., SUNDLER, F.: Peptidergic (Substance P) Nerves in the GenitoUrinary Tract. Neuroscience, 1978, 3, 419-425.

3. ALM, P.: On the autonomic innervation of the human vas deferens. Brain Re. Bull., 1982, 9, 673-677.

4. ALTHOF S.E. Pharmacologic treatment of rapid ejaculation, Psychiatric Clinics North Am. 1995

5. American Psychiatric Association. Diagnostic and statistical manual of mental disorders, third edition rev., Washington D.C., 1987

6. BARFIELD, R. J., WILSON, C. MCDONALD, P. G.: Sexual Behavior: Extreme Reduction of Postejaculatory Refractory Period by Midbrain Lesions in Male Rats. Science, 1975, 189: 147-149.

7. BAUM, M. J. EVERITT, B.J.: Increased expression of c-fos in the medial preoptic area after mating in male rats: Role of afferent inputs from the medial amygdala and midbrain central tegmental field. Neurosci., 1992, 50: 627-646.

8. BAUMGARTEN, H.G., HOLSTEIN, A.F., ROSENGREN, E.: Arrangement, ultrastructure and adrenergic innervation of smooth musculature of the ductuli efferentes, ductus epididymis and ductus deferens in man. Zeit. Zellforschung 1971, 120: 37-79.

9. BEACH, F. A.: Cerebral and hormonal control of reflexive mechanisms involved in copulatory behavior. Physiological Reviews, 1967, 47: 289-316.

10. BÉJIN A. L'orgasme chez l'homme adulte Andrologie, $1997,7: 336-342$

11. BELL, C.: Autonomic nervous control of reproduction: circulatory and other factors. Pharmacological Reviews, 1972, 24: 657-736.

12. BERGMAN, B., NILSSON, S. AND PETERSÉN, I.: The effect on erection and orgasm of cystectomy, prostatectomy and vesiculectomy for cancer of the bladder: a clinical and electromyographic study. British Journal of Urology, 1979, 51: 114-120.

13. BEYER, C., CONTERAS, J.L., LARSSON, K., OLMEDO, M. MORALI, G.: Patterns of motor and seminal vesicle activities during copulation in the male rat. Physiol. Behav. 1982, 29: 495-500.

14. BOHLEN, J. G., HELD, J. P. AND ANDERSON, M. $O .:$ Response of the circumvaginal musculature during masturbation. In Circumvaginal Musculature and Sexual Function. Edited by B. Graber. Basel:,18: 85- 94Karger, 1982, pp. 43-60.

15. BOHLEN, J. G., HELD, J. P. AND ANDERSON, M. O.: The male orgasm: pelvic contractions measured by anal probe. Archives of Sexual Behavior, 1980, 9: 503-521.

16. BORS, E. BLINN, K. A.: Bulbocavernosus reflex. Journal of Urology, 82: 128-130, 1959.

17. BORS, E. AND COMARR, A. E. Neurological disturbances of sexual function with special reference to 529 patients with spinal cord injury. Urological Survey, 1960, 10: 191-222. . 
18. BRACKETT, N. L. EDWARDS, D. A.: Medial preoptic connections with the midbrain tegmentum are essential for male sexual behavior. Physiology and Behavior, 1984, 32: 79-84.

19. BRACKETT, N. L., IUVONE, P. M. EDWARDS, D. A.: Midbrain lesions, dopamine and male sexual behavior. Behavioral Brain Research 1986, 20: 231240 .

20. BRINDLEY, G. S., GARDNER, B. P.: Sexual expression in paraplegia. Br. Med. J. 1991, 302: 113.

21. BRINDLEY, G. S.: Sexual and reproductive problems of paraplegic men. In Oxford Reviews of Reproductive Biology. Edited by J. R. Clarke. Oxford: Clarendon Press, 1986, pp. 214-222.

22. CALARESU, F. R. , MITCHELL, R.: Cutaneous mechanoreceptors in the glans penis of the rat. Brain Research, 1969, 15: 295-297.

23. CALARESU, F. R.: Experimental studies on the dorsal nerve of the penis of the rat. American Journal of Anatomy, 1970, 127: 415-421.

24. CARMICHAEL, M.S., WARBURTON, V.L., DIXEN, J. , DAVIDSON, J.M.: Relationships among cardiovascular, muscular and oxytocin responses during human sexual activity. Arch. Sex. Behav. 1994, 23: 59-79.

25. CHEN K.K., CHAN S.H.H., CHANG L.S., CHAN J.H.Y. Participation of paraventricular nucleus of hypothalamus in central regulation of penile erection in the rat J. Urol., 1997, $158: 238-234$

26. CLARK J. T.: Sexual function in altered physiological states: comparison of effects of hypertension, diabetes, hyperprolactinemia, and others to "normal" aging in male rats. Neuroscience \& Biobehavioral Reviews., 1995, 19: 279-302.

27. CLARK, T. K., CAGGIULA, A. R., MCCONNEL, R. A. ANTELMAN, S. M.: Sexual inhibition is reduced by rostral midbrain lesions in the male rat. Science, 1975, 190: 169-171.

28. COLLINS, W. F., ERICHSEN, J. T., ROSE, R. D.: Pudendal motor and premotor neurons in the male rat: A WGA transneuronal study. Journal of Comparative Neurology, 1991, 308: 28-41.

29. COLPI G.M., FANCIULACCI F., BERETTA G. NEGRI L., ZANOLO A., Evoked sacral potentials in subjects with true premature ejaculation Andrologia 1986, 583-586

30. CONTERAS, J.L. BEYER, C.: A polygraphic analysis of mounting and ejaculation in New Zealand white rabbit. Physiol. Behav., 1979, 23: 939-943.

31. COOLEN, L.M., PETERS, H.J.P.W. AND VEENING, J.G.: Fos immunoreactivity in the rat brain following consummatory elements of sexual behavior: a sex comparison. Brain Res., 1996, 738: 67-82.

32. DAIL, W.G.: Autonomic innervation of male reproductive organs. In Nervous control of the urogenital system. Edited by C.A. Maggi. Chur: Harwood Academic Press, 1993, 69-101.

33. DAVIDSON, J.M.: Activation of male rat's sexual behavior by intracerebral implantation of androgen. Endocrinol., 1966, 79: 783-794.

34. DE GROAT, W. C., BOOTH, A. M., YOSHI-MURA, N: Neurophysiology of micturition and its modification in animal models of human diseases. In Nervous control of the urogenital system. Edited by C.A. Maggi. Chur: Harwood Academic Press. , 1993, 227290

35. DE GROAT, W. C., BOOTH, A. M., MILNE, R. J. , ROPPOLO, J. R.: Parasympathetic preganglionic neurons in the sacral spinal cord. J. Auton. Nerv. Syst., 1982, 5: 23-43.

36. DE GROAT, W. C., NADELHAFT, I., MILNE, R. J., BOOTH, A. M., MORGAN, C., THOR, K.: Organization of the sacral parasympathetic pathways to the urinary bladder and large intestine. Journal of the Autonomic Nervous System, 1981, 3: 135-160.

37. DI SANT'AGNESE, P. A. DE MESY JENSEN, K. L.: Endocrine-paracrine [APUD). cells of the human female urethra and paraurethral ducts. Journal of Urology, 1987, 137: 1250-1254.

38. ECKHARD, C.: Untersuchungen über die erection des penis beim hunde. Beitrage zur Anatomie und Physiologie, 1863, 3: 123-150.

39. ELBADAWI, A. GOODMAN, D.C.: Autonomic innervation of accessory male genital glands. In Male accessory sex glands. Edited by E. Spring-Mills and E.S.E. Hafez. Holland: Elsevier, 1980, 101-128.

40. ELBADAWI, A., SCHENK, E.A.: The distribution of cholinergic and adrenergic nerves in the mammalian epididymis. A comparative histochemical study. Am. J. Anat., 1967, 121: 1-14.

41. ERTEKIN, C. REEL, F.: Bulbocavernosus reflex in normal men and in patients with neurogenic bladder and/or impotence. Journal of Neurological Science, 1976, 28: 1-15.

42. ERTEKIN C. ET AL., Hand and genital sympathetic skin potentials in flaccid and erectile penile states in normal potent men and patients with premature ejaculation, J. Urol., 1995, 153,76-79

43. EVERITT, B.J. STACEY, P.: Studies of instrumental behavior with sexual reinforcement in male rats. II: Effects of preoptic lesions, castration and testosterone. J. Comp. Psychol., 1987, 101: 407-419.

44. EVERITT, B.J., CADOR, M., ROBBINS, T.W.: Interactions between the amygdala and ventral striatum in stimulus-reward associations: Studies using a second-order schedule of sexual reinforcement. Neurosci., 1989, 30: 63-75.

45. FANCIULLACI F., COLPI G.M., BERETTA G., ZANOLO A., Cortical evoked potentials in subjects with true premature ejaculation Andrologia, 1988, 326-330 
46. FARRELL, J.I. , LYMAN, Y.: A study of the secretory nerves of, and the action of certain drugs on the prostate gland. Am. J. Physiol., 1937, 118: 64-70.

47. FEDAN, J.S., BESSE, J.C., CARPENTER, F.G. , TEAGUE, R.S.: Motor innervation of the smooth muscle of the rat seminal vesicle. J. Pharm. Exp. Ther., 1977, 201: 285-297.

48. FEDIRCHUK, B., SONG, L., DOWNIE, J. W., SHEFCHYK, S. J.: Spinal distribution of extracellular field potentials generated by electrical stimulation of pudendal and perineal afferents in the cat, Exp. Brain Res., 1992, 89: 517-520.

49. FRANK E., ANDERSON C., RUBINSTEIN D. Frequency of sexual dysfunction in normal couples $\mathrm{N}$ . Engl. J. Med. 1978, 299 : 111-115

50. GERSTENBERG, T. C., LEVIN, R. J. , WAGNER, G.: Erection and ejaculation in man. Assessment of the electromyography activity of the bulbocavernosus and ischiocavernosus muscles. British Journal of Urology, 1990, 65: 395-402.

51. GIULIANO F, RAMPIN O., BROWN K., COURTOIS F., BENOIT G., JARDIN A. Stimulation of the medial preoptic area of the hypothalamus elicits intracavernous pressure increases in the rat Neurosci. Let. $1996,209,1-4$

52. GODPODINOFF M. L. Journal of Sex and Marital Therapy 1989, 15: 130-134

53. GOODMAN R . E . The management of premature ejaculation The Journal of International Medical Research, 1977, 5 [suppl 1) 78-79

54. GOSLING, J. A.: Autonomic Innervation of the Prostate. In: Benign Prostatic Hypertrophy. Edited by F. Hinman, Jr. New York: Springer-Verlag, , 1983, 349-360.

55. GRAY, B. W., BECKETT, S. D., HENRY, D. F.: Microscopic characteristics of genital end bulbs in the penis of bulls. American Journal of Veterinary Research, 1985, 46: 2393-2398.

56. GREENBERG, J., SCHUBERT, W., METZ, J., YANAIHARA, N. FORSSMAN, W.-G.: Studies of the guinea pig epididymis. III. Innervation of epidydimal segments. 1985, Cell Tiss. Res. 239: 395-404.

57. Gross cited by Mario Luis Godpodinoff Journal of sex and Marital Therapy 1989, Vol 15, 130-134

58. HALATA, Z. MUNGER, B. L.: The neuroanatomical basis for the protopathic sensitivity of the human glans penis. Brain Research, 1986, 371: 205-230.

59. HALATA, Z., JOHNSON, R. D., KITCHELL, R. L. STRASSMANN, T.: The ultrastructure of sensory nerve endings in the penis of the goat. In: Mechanoreceptors. Edited by P. Hnik, T. Soucup, R. Vejsada and J. Zelena. New York: Plenum Press, 1988, 319-324.

60. HANCOCK, M. B. PEVETO, C. A.: A preganglionic autonomic nucleus in the dorsal gray commissure of the lumbar spinal cord of the rat. Journal of Comparative Neurology, 1979, 183: 65-72.

61. HANCOCK, M. B., PEVETO, C. A.: Preganglionic neurons in the sacral spinal cord of the rat: An HRP study. Neuroscience Letters, 1979, 11: 1-5.

62. HEAD, H.: Studies in neurology. London: Oxford University Press, 1920.

63. HEIMER, L. LARSSON, K.: Impairment of mating behavior in male rats following lesions in the preoptic-anterior hypothalamic continuum. Brain Research, 1966,3: 248-263.

64. HERBERG, L. J.: Seminal Ejaculation Following Positively Reinforcing Electrical Stimulation of the Rat Hypothalamus. J. Comp. Physiol. Psychol. , 1963, 56: 679-685.

65. HIGGINS, J.R.A. , GOSLING, J.A.: Studies on the structure and intrinsic innervation of the normal human prostate. The Prostate, Suppl. 2: 5-16, 1989.

66. HODSON, N.: The role of the hypogastric nerves in seminal emission in the rabbit. J. Reprod. Fert. 1964, 7: 113-122.

67. HOLMES, G. M. , SACHS, B. D.: The ejaculatory reflex in copulating rats: normal bulbospongiosus activity without urethral stimulation. Neuroscience Letters, 1991, 125: 195-197.

68. HONDA, C. N. LEE, C. L.: Immunohistoche-mistry of synaptic input and functional characterizations of neurons near the spinal central canal. Brain Research , 1985, 343: 120-128.

69. HONDA, C. N.: Visceral and somatic afferent convergence onto neurons near the central canal in the sacral spinal cord of the cat Journal of Neurophysiology, 1985, 11: 1059-1076.

70. HONDA, C. N. PERL, E.: Functional and morphological features of neurons in the midline region of the caudal spinal cord of the cat. Brain Research , 1985, 340: 285-295.

71. HULL, E. M., BITRAN, D., PEHEK, E. A., WARNER, R. K., BAND, L. C. HOLMES, G. M.: Dopaminergic control of male sex behavior in rats: Effects of an intracerebrally-infused agonist. Brain Research , 1986, 370: 73-81.

72. JÄNIG, W. MCLACHLAN, E. M.: Organization of lumbar spinal outflow to distal colon and pelvic organs. Physiological Reviews, 1987, 67: 1332-1403.

73. JOHNSON, R. D. DUGAN, V. P.: Responses of spine associated penile mechanoreceptors to tangential mechanical stimulation. Society for Neuroscience Abstracts, 1988, 14: 726.

74. JOHNSON, R. D., HALATA, Z.: Topography and ultrastructure of sensory nerve endings in the glans penis of the rat. Journal of Comparative Neurology, 1991, 312: 299-310.

75. JOHNSON, R. D. KITCHELL, R. L.: Mechanoreceptor response to mechanical and ther- 
mal stimuli in the glans penis of the dog. Journal of Neurophysiology, 1987, 57: 1813-1836.

76. JOHNSON, R. D.: Efferent modulation of penile mechanoreceptor activity. Progress in Brain Research, 1988, 74: 319-324.

77. JOHNSON, R. D.: Physiology of single spinal cord neurons responding to penile stimulation in the rat. Society for Neuroscience Abstracts, 1989, 15: 756.

78. JOHNSON, R. D.: Inhibitory role of mechanoreceptive afferents from the distal glans penis on spinal sexual reflexes in the rat. Society for Neuroscience Abstracts, 1990, 16: 562.

79. KAPLAN H .S., The New Sex Therapy New York Brunner, Mazel Publishers 1974, 289-315

80. KHUR C.S., HEIMAN J., CAEDENAS D., BRADLEY W., BERGER R.E. Premature emission after spinal cord injury J. Urol., 1995, $153: 429-431$

81. KIMURA Y. ADACHI K. KISAKI N. ISE K.: On the transportation of spermatozoa in the vas deferens. Andrologia., 1975, 7: 55-61.

82. KIMURA Y. ADACHI K. KISAKI N.: ISE K. Role of alpha-adrenergic receptor mechanism in closure of the internal urethral orifice during ejaculation. Urologia Internationalis., 1975, 30: 341-9.

83. KIMURA, Y.: On peripheral nerves controlling ejaculation. Tohoku Journal of Experimental Medicine, 105: 177-190, 1970.

84. KINSEY D.C. Sexual Behavior in the human male Phildelphia, Saunders, 1948

85. KINSEY, A. C., POMEROY, W. B., MARTIN, C. E. GEBHARD, P. H.: Sexual Behavior in the Human Female. Philadelphia: W. B. Saunders, 1953.

86. KITCHELL, R. L., GILANPOUR, H. JOHNSON, R. D.: Electrophysiologic studies of penile mechanoreceptors in the rat. Experimental Neurology, 1982, 75: 229-244.

87. KLUVER, H. BUCY, P.C.: Preliminary analysis of functions of the temporal lobes in monkeys. Arch. Neurol. Psychiat., 1939, 42: 979-1000.

88. KOLBECK, S. C. STEERS, W. D.: Neural regulation of the vas deferens in the rat: An electrophysiological analysis. Am. J. Physiol. Regul. Integr. Comp. Physiol., 1992, 263: R331-R338.

89. KOLLBERG, S., PETERSEN, I. STENER, I.: Preliminary results of an electromyographic study of ejaculation. Acta Chirurgica Scandinavica, 1962, 123: 478-483.

90. KUZUHARA, S., KANAZAWA, I. NAKANISHI, T.: Topographical localization of the Onuf's nuclear neurons innervating the rectal and vesical striated sphincter muscles: a retrograde fluorescent double labeling in cat and dog. Neurosci. Lett. 1980, 16: 125130.

91. LAMANO CARVALHO, T.L., HODSON, M.P.,
BLANK, M.A., WATSON, P.F., MULDERRY, P.K., BISHOP, A.E., BLOOM, S.R. POLAK, J.M.: Occurrence, distribution and origin of peptide-containing nerves of guinea-pig and rat male genitalia and the effects of denervation on sperm characteristics. J. Anat., 1986, 149: 121-141.

92. LANGLEY, J. N. , ANDERSON, H. K.: The innervation of the pelvic and adjoining viscera. Part III. The external generative organs. Journal of Physiology, 1895, 19: 85-121.

93. LANGLEY, J. N. ANDERSON, H. K.: The innervation of the pelvic and adjoining viscera. Part IV. The internal generative organs. Journal of Physiology, 1895, 19: $122-130$.

94. LAVOISIER, P., PROULX, J., COURTOIS, F., DECARUFEL, F. DURAND, L. -G.: Relationship between perineal muscle contractions, penile tumescence, and penile rigidity during nocturnal erections. Journal of Urology, 1988, 139: 176-179.

95. LAVOISIER, P., COURTOIS, F., BARRES, D. BLANCHARD, M.: Correlation between intracavernous pressure and contraction of the ischiocavernosus muscle in man. Journal of Urology, 1986, 136 : 936-939.

96. LAVOISIER, P., PROULX, J. COURTOIS, F.: Reflex contractions of the ischiocavernosus muscles following electrical and pressure stimulations. Journal of Urology, 1988, 139: 396-399.

97. LINCOLN, J. BURNSTOCK, G.: Autonomic innervation of the urinary bladder and urethra. In Nervous control of the urogenital system. Edited by C.A. Maggi. Chur: Harwood Academic Press, 1993. 33-68.

98. MAGGI, C.A., SANTICIOLI, P., THEODORSSONNORHEIM, E. MELI, A.: Immunoblockade of response to capsaicin in the rat vas deferens: Evidence for the involvement of endogenous calcitonin generelated peptide. Neurosci. Lett., 1987, 78: 63-68.

99. MAGGI, C.A.: The role of peptides in the regulation of micturition reflex: An update. Gen. Pharm., 1991, 22: $1-4$.

100. MALSBURY, C. W.: Facilitation of male rat copulatory behavior by electrical stimulation of the medial preoptic area. Physiology and Behavior, 1971, 7: 797805, .

101. MARBERGER, H.: Mechanisms of ejaculation. In: Physiology and Genetics of Reproduction. Edited by E. M. Coutinho and F. Fuchs. New York and London: Plenum Press, 1974, 99-110.

102. MARSON, L. MCKENNA, K. E.: A role for 5hydroxytryptamine in mediating spinal sexual reflexes, Experimental Brain Research, 1992, 88: 313-320.

103. MARSON, L. MCKENNA, K. E.: Stimulation of the hypothalamus initiates the urethrogenital reflex in male rats Brain Res., 1994, 638: 103-108.

104. MARSON, L. , MCKENNA, K. E.: The identification 
of a brainstem site controlling spinal sexual reflexes in male rats. Brain Research, 1990, 515: 303-308.

105. MARSON, L., MCKENNA, K.E.: CNS cell groups involved in the control of the ischiocavernosus and bulbospongiosus muscles: A transneuronal tracing study using pseudorabies virus, Journal of Comparative Neurology,1996, 374: 161-179.

106. MARSON, L. , ORR, R.: Identification of rat spinal neurons that innervate the prostate: comparison of hypogastric and pelvic inputs using transneuronal tracing with pseudorabies virus. Society for Neuroscience Abstracts, 1996, 26: 215.

107. MARSON, L., PLATT, K. B., MCKENNA, K. E.: Central nervous system innervation of the penis as revealed by the transneuronal transport of pseudorabies virus. Neuroscience, 1993, 55: 263-280.

108. MARSON. L., LIST, M., MCKENNA, K. E.: Lesions of the nucleus paragigantocellularis alter ex copula penile reflexes. Brain Research, 1992, 592: 187-192.

109. MASTERS, W. H. , JOHNSON, V. E.: Human Sexual Response. Boston: Little, Brown and Co., 1966

110. MASTERS W.H. , JOHNSONV.E. in Human Sexual Inadequacy , Boston, Little, Brown Company 1970, $92-115$

111. MCKENNA, K. E. , NADELHAFT, I.: The organization of the pudendal nerve in the male and female rat. Journal of Comparative Neurology, 248: 532$549,1986$.

112. MCKENNA, K. E., NADELHAFT, I.: The pudendopudendal reflex in male and female rats. Journal of the Autonomic Nervous System, 1989, 27: 67-77.

113. MCKENNA, K. E., CHUNG, S. K. , MCVARY, K. T.: A model for the study of sexual function in anesthetized male and female rats. American Journal of Physiology, 1991, 261: R1276-1285.

114. MCKENNA, K. E., KNIGHT, K., MAYERS, R.: Modulation of the threshold for sexual reflexes in female rats by peripheral serotonin. Pharmacology, Biochemistry and Behavior, 1991, 40: 151-156.

115. MCKENNA K.E., GIULIANO F., RAMPIN O., BERNABE J. Electrical stimulation of the paraventricular nucleus induces penile erection and ejaculation in the rat Soc. Neurosci. Abstr, 1997

116. MCVARY, K. T., RAZZAQ, A., LEE, C., VENEGAS, M. F., RADEMAKER, A., MCKENNA, K. E.: Growth of the rat prostate gland is facilitated by the autonomic nervous system. Biol. Reprod., 1994, 51: 99-107.

117. MELDRUM, L.A. , BURNSTOCK, G.: Evidence that ATP acts as a cotransmitter with noradrenaline in sympathetic nerves supplying the guinea-pig vas deferens. Eur. J. Pharmacol., 1983, 92: 161-163.

118. MELDRUM, L.A., BURNSTOCK, G.: Evidence that ATP is involved as a cotransmitter in the hypogastric nerve supplying the seminal vesicle of the guinea-pig. Eur. J. Pharmacol., 1985, 110: 363-366.
119. MERARI, A., GINTON, A.: Characteristics of exaggerated sexual behavior induced by electrical stimulation of the medial preoptic area in males rats. Brain Res., 1975, 86: 97-108.

120. MORGAN, C., DEGROAT, W.C. , NADELHAFT, I.: The spinal distribution of sympathetic preganglionic and visceral primary afferent neurons that send axons into the hypogastric nerves of the cat. J. Comp. Neurol., 1986, 243: 23-40.

121. MORGAN, C., NADELHAFT, I., DE GROAT, W.C.: The distribution of visceral primary afferents from the pelvic nerve to Lissauer's tract and the spinal gray matter and its relationship to the sacral parasympathetic nucleus. J. Comp. Neurol. 1981, 201: 415-440.

122. NADELHAFT, I., BOOTH, A. M.: The location and morphology of preganglionic neurons and the distribution of visceral afferents from the rat pelvic nerve: a horseradish peroxidase study. Journal of Comparative Neurology, 1984, 226: 238-245.

123. NADELHAFT, I., MCKENNA, K. E.: Immu-nocytochemical localization of transported wheat germ agglutinin in the spinal cord of the rat. Society for Neuroscience Abstracts, 1983, 9: 258.

124. NADELHAFT, I., MCKENNA, K. E.: Sexual dimorphism in sympathetic preganglionic neurons of the rat hypogastric nerve. Journal of Comparative Neurology, 1987, 256: 308-315.

125. NADELHAFT, I., DE GROAT, W.C., MORGAN C.: Location and morphology of parasympathetic preganglionic neurons in the sacral spinal cord of the cat revealed by retrograde axonal transport of horseradish peroxidase. J. Comp. Neurol. 1980, 193: 265-281.

126. NAKANISHI, H., TAKEDA, H.: The possible role of adenosine triphosphate in chemical transmission between the hypogastric nerve terminal and seminal vesicle in the guinea-pig. Jap. J. Pharmacol., 1973, 23: $479-490$.

127. National Institute of Health Consensus development conference statement on impotence Int. J. Impotence Res.1993, 5 :181-284

128. NUÑEZ, R., GROSS, G. H., SACHS, B. D.: Origin and central projections of rat dorsal penile nerve: possible direct projection to autonomic and somatic neurons by primary afferents of nonmuscle origin. J. Comp. Neurol., 1986, 247: 417-429.

129. PATRIZI, G. , MUNGER, B.L.: The cytology of encapsulated nerve endings in the rat penis. J. Ultrastruct. Res., 1965, 13: 500-515.

130. PESCATORI, E. S., CALABRO, A., ARTIBANI, W., PAGANO, F., TRIBAN, C., ITALIANO, G.: Electrical stimulation of the dorsal nerve of the penis evokes reflex tonic erections of the penile body and reflex ejaculatory responses in the spinal rat. Journal of Urology., 1993., 149: 627-632 
131. PESHORI, K. R., ERICHSEN, J. T. , COLLINS, W. F.: Differences in the connectivity of rat pudendal motor nuclei as revealed by retrograde transneuronal transport of wheat germ agglutinin. Journal of Comparative Neurology , 1995, 353: 119-128.

132. PETERSÉN, I., STENER, I.: An electromyographical study of the striated urethral sphincter, the striated anal sphincter, and the levator ani muscle during ejaculation. Electromyography, 1970, 1: 2368.

133. RAMPIN O.,GOUGIS S.,GIULIANO F,,ROUSSEAU J.P .Spinal C-fos labelling and penile erection elicited by stimulationof the dorsal nerve of the penis in the rat Am. J. Physiol. 1997, 272 : R1425- R1431

134. RAND, M. J.: Nitrergic transmission: Nitric oxide as a mediator of non-adrenergic, non-cholinergic neuroeffector transmission. Clin. Exp. Pharmacol. Physiol., 1992, 19: 147-169.

135. RATTNER, W. H., GERLAUGH, R. L., MURPHY, J. J., ERDMAN, W. J., II: The bulbocavernosus reflex. I. Electromyographic study of normal patients. Journal of Urology, 1958, 80: 140-141.

136. RECKER, F. TSCHOLL, R.: Monitoring of emission as direct intraoperative control for nerve sparing retroperitoneal lymphadenectomy. Journal of Urology., 1993, 150: 1360-1364.

137. RECKER, F., GOEPEL, M., OTTO, T., KREGE, S., WERNLI, M., STUCKI, P., TSCHOLL, R., RUBBEN, H.: An intra-operative seminal and prostate emission test as a control for nerve-sparing procedures in primary and secondary retroperitoneal lymphadenectomy. British Journal of Urology., 1996, 77: 133-137.

138. ROBINSON, B.W., MISHKIN, M.: Ejaculation evoked by stimulation of the preoptic area in monkeys. Physiol. Behav., 1966, 1: 269-272.

139. ROPPOLO, J. R., NADELHAFT, I., DE GROAT, W. C.: The organization of pudendal motoneurons and primary afferent projections in the spinal cord of therheusmonkey revealed by horseradish peroxidase. J. Comp. Neurol., 1995, 234:475-488.

140. ROSE, J. D.: Forebrain influences on brainstem and spinal mechanisms of copulatory behavior: A current perspective on Frank Beach's contribution. Neurosci. Biobehav. Rev. , 1990, 14: 207-215.

141. ROWLAND D.L., HAENSEL S.M., BLOM J.H., SLOB A. K. Penile sensitivity in men with premature ejaculation and erectile dysfunction J. Marital Ther. 1993, $19: 189$

142. ROWLAND D.L., SLOB A.K. Understanding and diagnosing sexual dysfunction : recent progress through psychophysiological and psychophysical methods Neurosci. Biobehav. Rev. 1995, 19 : 201-209

143. SAPER, C. B., LOEWY, A. D., SWANSON, L. W. COWAN, W. M.: Direct hypothalamo-autonomic connections. Brain Research, 1976, 117: 305-312.
144. SAR, M. AND STUMPF, W. E.: Distribution of androgen concentrating neurons in the rat brain. In: Anatomical Neuroendocrinology. Edited by W. E. Stumpf and L. D. Grant. Karger, Basel, 1975. 120$133,$.

145. SARREL L .J ., SARREL P.M. Sexual turning points: the seven stage of adult sexuality New York Mac Millan 1984: 11-7.

146. SCHMIDT, M. H., SCHMIDT, H. S.: The ischiocavernosus and bulbospongiosus muscles in mammalian penile rigidity Sleep 16: 171-183.

147. SCHRøDER, H. D.: Anatomical and pathoanatomical studies on the spinal efferent systems innervating pelvic structures. Journal of the Autonomic Nervous System, 1985, 14: 23-48.

148. SETCHELL, B.P., BROOKS, D.E.: Anatomy, vasculature, innervation, and fluids of the male reproductive tract. In: The Physiology of Reproduction, edited by E. Knobil and J. Neill. New York: Raven Press, 1988, 753-836.

149. SHIMURA, T., SHIMOKOCHI, M.: Modifi-cation of male rat copulatory behavior by lateral midbrain stimulation. Physiology and Behavior 1991; 50: 989994.

150. SIMERLY, R. B., SWANSON, L. W.: Neuro-transmitter specificity of cells and fibers in the medial preoptic nucleus: An immunohistochemical study in the rat. Journal of Comparative Neurology, 1986, 246: 343-363.

151. SIMERLY, R. B., SWANSON, L. W.: Projections of the medial preoptic nucleus: Phaseolus vulgaris leucoagglutinin anterograde tract-tracing study in the rat. Journal of Comparative Neurology, 1988, 270: 209-242.

152. SIMERLY, R. B., SWANSON, L. W.: The organization of neural inputs to the medial preoptic nucleus of the rat. Journal of Comparative Neurology, 1986, 246: 312-342.

153. SJÖSTRAND, N. O.: The adrenergic innervation of the vas deferens and the accessory male genital glands. Acta Physiologica Scandinavica, 1965, 65: 581 .

154. SLIMP, J.C., HART, B.L., GOY, R.W.: Heterosexual, autosexual and social behavior of adult male rhesus monkeys with medial preoptic-anterior hypothalamic lesions. Brain Res. 1975, 142: 105-122,

155. SMITH, E. R. , LEBEAUX, M.: The composition of nerve induced canine prostatic secretion. Invest Urol, 1971; 9: 100-103

156. SMITH, E. R.: The Canine Prostate and Its Secretion. In: Molecular Mechanisms of Gonadal Hormone Action. Edited by J. A. Thomas and R. L. Singhal. Baltimore: University Park Press,1975, 167-204.

157. STJERNQUIST, M., HAKANSON, R., LEANDER, S., OWMAN, C., SUNDLER, F. , UDDMAN, R.: 
Immunohistoche-mical localization of substance $P$, vasoactive intestinal polypeptide and gastrin-releasing peptide in vas deferens and seminal vesicle, and the effect of these and eight other neuropeptides on resting tension and neurally evoked contractile activity. Regul. Peptides, 1983, 7: 67-86.

158. STJERNQUIST, M., OWMAN, C., SJOBERG, N. O., SUNDLER, F.: Coexistence and cooperation between neuropeptide $Y$ and norepinephrine in nerve fibers of guinea pig vas deferens and seminal vesicle. Biol. Reprod., 1987, 36: 149-155.

159. STRASSBERG D.S., KELLY M.P., CARLL C., KIRCHER J.C. The psychophysiological nature of in premature ejaculation Arch. Sex. Behav. 1987, 16 : 327-336

160. STRASSBERG D.S., MAHONEY J.M., SCHAUGAARD M., HALE V.E. The role of anxiety in premature ejaculation : a psychophysiological model Arch. Sex. Behav. 1990, 19 : 251-257

161. SWANSON, L.W., SAWCHENKO, P.E.: Hypothalamic integration: Organization of the paraventricular and supraoptic nuclei. Ann. Rev. Neurosci., 1983, 6: 269-324.

162. TANG Y.,RAMPIN O.,CALAS A.,FACCHINETTI P.,GIULIANO F. Oxytocinergic and serotoninergic innervation of the identified lumbosacral nuclei controlling penile erection in the male rat Neuroscience 1998,1 : 241-254

163. TERASAKI, T.: Effects of autonomic drugs on intraluminal pressure and excretion of rat seminal vesicles in vivo. Tohoku J. Exp. Biol. Med., 1989, 157: $373-379$.

164. THOR, K.B., MORGAN, C., NADELHAFT, I., HOUSTON, M., DE GROAT, W.C.: Organization of afferent and efferent pathways in the pudendal nerve of the female cat. J. Comp. Neurol. , 1989, 288: 263-279.

165. UEYAMA, T., ARAKAWA, H., MIZUNO, N.: Central distribution of efferent and afferent components of the pudendal nerve in rat. Anatomy and Embryology, 1987, 177: 37-49.

166. VAALASTI, A., HERVONEN, A.: Autonomic innervation of the human prostate. Invest. Urol. 1980, 17: 293-297.

167. VAALASTI, A., HERVONEN, A.: Nerve endings in the human prostate. Am. J. Anat., 1980, 157: 41-47.

168. VAALASTI, A., LINNOILA, I., HERVONEN, A.: Immunohistochemical demonstration of VIP, [Met5]and [Leu5]-enkephalin immunoreactive nerve fibres in the human prostate and seminal vesicles. Histochem., 1980, 66: 89-98.

169. VAN DIS, H., LARSSON, K.: Seminal discharge following intracranial electrical stimulation. Brain Research, 1970, 23: 381-386.
170. VIZZARD, M. A., ERICKSON, V. L., CARD, J. P., ROPPOLO, J. R., DE GROAT, W. C.: Transneuronal labeling of neurons in the adult rat brainstem and spinal cord after injection of pseudorabies virus into the urethra J. Comp. Neurol., 1995, 355: 629-640.

171. WABREK, A. J.: Bulbocavernosus reflex testing in 100 consecutive case of erectile dysfunction. Urology,, 1985., 25: 495-498

172. WAGNER, C. K., CLEMENS, L. G.: Projections of the paraventricular nucleus of the hypothalamus to the sexually dimorphic lumbosacral region of the spinal cord. Brain Research, 1991, 539: 254-262.

173. WANG, J. -M., MCKENNA, K. E., LEE, C.: Determination of prostatic secretion in rats: effect of neurotransmitters and testosterone. The Prostate, 1991, 18: 289-301.

174. WANG, J.-M., K.E. MCKENNA, K.T. MCVARY, C. LEE. Requirement of innervation for maintenance of structural and functional integrity in the rat prostate. Biol. of Reprod., 1991, 44: 1171-1176.

175. WATANABE, H. , YAMAMOTO, T.: Autonomic innervation of the muscles in the wall of the bladder and proximal urethra of male rats. J. Anat., 1979, 128: 873-886.

176. WATANABE, H., SHIMA, M., KOJIMA, M., OHE, H.: Dynamic study of nervous control on prostatic contraction and fluid excretion in the dog. J. Urol. , 1988, 140: 1567-1570.

177. WHITELAW, G.P., SMITHWICK, R.H.: Some secondary effects of sympathectomy with particular reference to disturbance of sexual function. New Eng. J. Med., 1951, 245: 121-130.

178. XIN ET AL. Penile sentivity in patients with primary premature ejaculation J. Urol.1996, 156 :979-981

179. YAMAUCHI, A. , BURNSTOCK, G.: Post-natal development of the innervation of the mouse vas deferens: A fine structural study. J. Anat., 1969, 104: 17-32.

180. YANIGIMOTO, M., HONDA, K., GOTO, Y., NEGORO, H.: Afferents originating from the dorsal penile nerve excite oxytocin cells in the hypothalamic paraventricular nucleus of the rat. Brain Res., 1996, 733: 292-296.

181. YELLS, D. P., HENDRICKS, S. E., PRENDERGAST, M. A.: Lesions of the nucleus paragigantocellularis: Effects on mating behavior in male rats. Brain Research. , 1992, 596: 73-79.

182. YURI, K. Immunohistochemical and enzyme histochemical localization of peptidergic, aminergic and cholinergic nerve fibers in the rat seminal vesicle. J. Urol. , 1990, 143: 194-198. 


\begin{abstract}
Rationale for a pathophysiological approach of ejaculatory disturbances

F. Giuliano, K. Mac Kenna, S. Droupy, O. RAMPIN, V. IZARD, G. BENOIT, A. JARDIN
\end{abstract}

Seminal emission and sperm expulsion are under the control of both the sympathetic and parasympathetic outflows and also of the somatic innervation conveyed by the pudendal nerve. The 2 phases of ejaculation are reflexive with the reflexes handled at the thoraco-lumbar and sacral levels of the spinal cord. Such a spinal organization remains widely unknown. The role of various peripheral neurotransmitters has been evidenced including norepinephrine and acetylcholine and also peptidergic, purinergic i.e. ATP and nitric oxide. Stimulation of the seminal tract afferents play a crucial in the onset of ejaculatory mechanisms. Except for the dorsal nerve of the penis, there is a lack of information concerning these afferents. Several supraspinal centers i.e. hypothalamus, medial amygdala, pons and nucleus paragigantocellularis exert descending and ascending inhibitory and excitatory influences on spinal nuclei controlling emission and expulsion of sperm. Central neurotransmission responsible for this supraspinal control could involve serotonin, oxytocin and norepinephrine. In the light of the available anatomical and neurophysiological data, pathophysiological aspects of ejaculatory disorders are futher discussed. Premature ejaculation could be related to a periheral and central hypersentivity. Most of the other ejaculation abnormalities are likely mainly related to an impairment of the central mechanisms.

Key-words : Ejaculation, emission, neurophysiology, central nervous system, premature ejaculation 